\title{
Occupational Hazard: Radiation Exposure for the Urologist - Developing a Reference Standard
}

\author{
Seth A. Cohen, Sriram S. Rangarajan, Tony Chen, Kerrin L. Palazzi, J. Scott Langford, Roger L. Sur \\ Department of Surgery and Division of Urology, U C San Diego Health Science System, San Diego, CA.
}

\section{ABSTRACT}

Introduction: To date, there is a paucity of literature offering practicing urologists a reference for the amount of radiation exposure received while surgically managing urolithiasis. This study examines the cumulative radiation exposure of an urologist over 9 months. Materials and Methods: We present a case series of fluoroscopic exposures of an experienced stone surgeon operating at an academic comprehensive stone center between April and December 2011. Radiation exposure measurements were determined by a thermoluminescent dosimeter worn on the outside of the surgeon's thyroid shield. Estimations of radiation exposure (mrem) per month were charted with fluoroscopy times, using scatter plots to estimate Spearman's rank correlation coefficients.

Results: The total 9-month radiation exposure was 87 mrems for deep dose equivalent (DDE), 293 mrem for lens dose equivalent (LDE), and 282 mrem for shallow dose equivalent (SDE). Total fluoroscopy time was 252.44 minutes for 64 ureteroscopies (URSs), 29 percutaneous nephrolithtomies (PNLs), 20 cystoscopies with ureteral stent placements, 9 shock wave lithotripsies (SWLs), 9 retrograde pyelograms (RPGs), 2 endoureterotomies, and 1 ureteral balloon dilation. Spearman's rank correlation coefficients examining the association between fluoroscopy time and radiation exposure were not significant for DDE ( $p=0.6$, Spearman's rho $=0.2$ ), LDE $(p=0.6$, Spearman's rho $=0.2)$, or SDE $(p=$ 0.6 , Spearman's rho $=0.2$ ).

Conclusions: Over a 9-month period, total radiation exposures were well below annual accepted limits (DDE 5000 mrem, LDE 15,000 mrem and SDE 50,000 mrem). Although fluoroscopy time did not correlate with radiation exposure, future prospective studies can account for co-variates such as patient obesity and urologist distance from radiation source.

\section{ARTICLE INFO}

\section{Key words:}

Urolithiasis; Nephrolithiasis;

Kidney Calculi; Radiation

Injuries; Urologic Surgical

Procedures; Neoplasms

Int Braz J Urol. 2013; 39: 209-13

Submitted for publication:

September 03, 2012

Accepted after revision:

January 30, 2013

\section{INTRODUCTION}

Recent literature has introduced the risks of radiation exposure for patients. Studies have found a $600 \%$ increase in medical radiation exposure to the United States (U.S.) population since 1980 (1). Given the importance of imaging to kidney stone diagnosis and treatment, efforts have been made to standardize recommendations in order to balance radiographic imaging with its inherent long term risks, such as those seen with repeat computerized tomography (CT) for nephrolithiasis (2). Parallel movements have occurred to efficiently use fluoroscopy in the operating room to decrease patient exposure (3-5).

Although understanding patient radiation exposure risks is clearly critical, urologic health care worker exposure has also been investigated recently to determine risks in the work environment (6). In a 2011 survey sent to members of the Endourological Society, compliance with chest 
and pelvic shields was reported to be $97 \%$; however, usage of thyroid shields, dosimeters, lead-impregnated glasses, and gloves were only 68\%, 34.3\%, 17.2\%, and 9.7\% respectively (7). Most reports of urologic health care worker radiation exposure risks include data on procedure-specific radiation scatter, i.e., how much radiation scatter occurs during an average ureteroscopy or percutaneous case. The current literature lacks data on long-term radiation exposure that urologists receive for all "general" endourologic cases. This study examines the cumulative radiation exposure of an urologist over 9 months, taking into account radiation exposure for all endourologic procedures [ureteroscopy (URS), shock wave lithotripsy (SWL), percutaneous nephrolithotomy (PNL), cystoscopy, retrograde pyelograms, etc.].

\section{MATERIALS AND METHODS}

We retrospectively analyzed data from our Institutional Review Board-approved database documenting a case series of fluoroscopic exposures of a single right-handed, experienced stone surgeon operating at an academic comprehensive kidney stone center. A waiver of consent was obtained as the study presented no more than minimal risk to human subjects and involved no procedures for which written consent was normally required, outside of the context of the investigation. All cases utilizing fluoroscopy between April and December 2011 were included in the dataset. Radiation exposure measurements were determined by a single thermoluminescent dosimeter (TLD) worn on the outside of the surgeon's thyroid shield. All fluoroscopic imaging was performed with one of two available under-couch X-ray emitter and over-couch image intensifiers (GE OEC 9800 \&t 9900). The urologist wore a $0.5 \mathrm{~mm}$ lead thyroid shield, lead apron, and lead-impregnated glasses during all endourologic procedures requiring fluoroscopy. Radiation exposure for both lens dose equivalent (LDE) and shallow dose equivalent (SDE) were obtained directly from the single TLD. To account for lead being worn, TLD readings were multiplied by 0.3 to yield deep dose equivalent (DDE) radiation exposure values. All readings were expressed in millirem (mrem) which is one-thousandth of a rem (Roentgen equivalent man). The monthly fluoroscopy times for all surgeries were recorded as well. Estimations of radiation exposure (mrem) per month were then charted with fluoroscopy times, using scatter plots to estimate Spearman's rank correlation coefficients with Type I error alpha $=0.05$.

\section{RESULTS}

A total of 137 surgical procedures using fluoroscopy were identified over this 9-month period. Complete fluoroscopy time data was available for 134 procedures; 3 procedures without complete fluoroscopy time data were excluded from analysis. The total 9-month radiation exposure was 87 mrems for deep dose equivalent (DDE), $293 \mathrm{mrem}$ for lens dose equivalent (LDE), and $282 \mathrm{mrem}$ for shallow dose equivalent (SDE). Total fluoroscopy time during this period was 252.44 minutes for: 64 URS, 29 PNL, 20 cystoscopies with ureteral stent placements, 9 SWL, 9 RPGs, 2 endoureterotomies, and 1 ureteral balloon dilation (Table- 1 and Figure-1). Spearman's rank correlation coefficients examining the association between fluoroscopy time and radiation exposure were not significant for DDE ( $\mathrm{p}=0.6$, Spearman's rho $=$ $0.2)$, LDE ( $p=0.6$, Spearman's rho $=0.2$ ), or SDE $(p=0.6$, Spearman's rho $=0.2)$.

\section{DISCUSSION}

The risks posed by a urological career's worth of low-dose ionizing radiation to practicing surgeons remain unclear (8). The 2007 International Commission on Radiation Protection (ICRP) guidelines recommend an occupational dose limit of no more than $50 \mathrm{mSv}$ $(5,000 \mathrm{mrem})$ per year or more than $100 \mathrm{mSv}(10,000$ mrem) averaged over 5 years (9). U.S. regulations (Title 10, part 20 of the Code of Federal Regulations) mandate annual accepted limits (DDE 5000 mrem, LDE 15,000 mrem and SDE 50,000 mrem). To ensure practitioners are within these guidelines, there exists a need for a controlled measurement of radiation exposure experienced by practicing U.S. urologists over a period of time - an aim that formed the basis of this study.

The applicability of recent literature looking at radiation exposure to urologists has been limited 
Table 1 - Cumulative Radiation Exposure and Time.

\begin{tabular}{lcccc}
\hline Month & Time (min.) & DDE (mrem) & LDE (mrem) & SDE (mrem) \\
\hline April & 30.49 & 9 & 31 & 30 \\
May & 43.09 & 5 & 17 & 16 \\
June & 17.04 & 0 & 0 & 0 \\
July & 18.35 & 4 & 14 & 13 \\
August & 39.01 & 7 & 22 & 22 \\
September & 36.39 & 15 & 51 & 51 \\
October & 38.53 & 21 & 70 & 66 \\
November & 11.16 & 12 & 41 & 38 \\
December & 18.38 & 14 & 47 & 46 \\
\hline Sum & $\mathbf{2 5 2 . 4 4}$ & $\mathbf{8 7}$ & $\mathbf{2 9 3}$ & $\mathbf{2 8 2}$ \\
\hline
\end{tabular}

Figure 1 - Endourological Surgeries Requiring Fluoroscopy Over 9 Months.

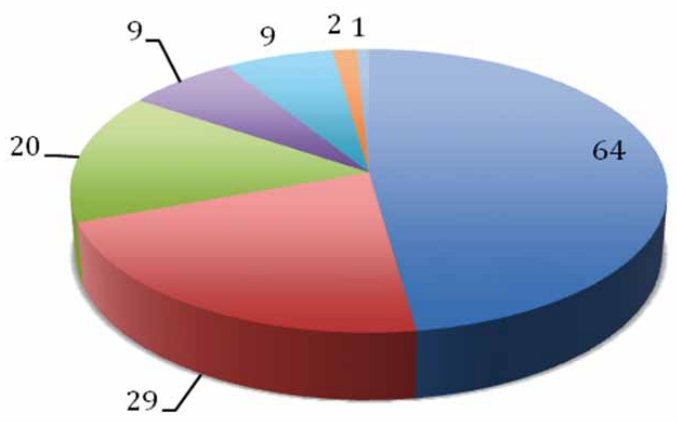

by two factors: every major study from the past 25 years has been 1) conducted abroad and/or 2) focused specifically on the radiation doses of individual procedures, particularly PNL $(6,10-13)$. The most recent exposure data from North American institutions occurred in the distant past: a 1986 radiation exposure report of 7 PNLs and a 1996 radiation exposure report of 5 unspecified urologic procedures while analyzing the efficacy of a newly designed fluoroscopic drape $(14,15)$.

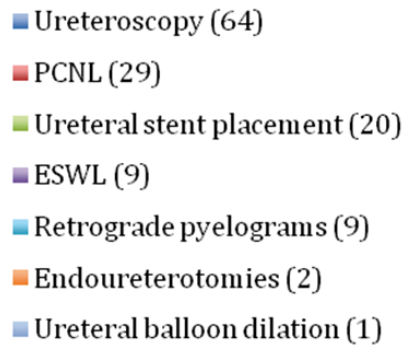

Most recently, a German study reported data of 235 pooled and averaged dose exposure TLD readings from five different urologic procedures performed by 12 surgeons over 6 months (16). While radiation exposure data from European institutions have contributed to our understanding of the radiation risks faced by urologists, inherent characteristics of these investigations make it difficult for urologists in North America to generalize the data to their environments. 
For example, exposures may differ secondary to varying practice patterns among international countries versus those in North America. This may be secondary to differences in training and equipment, but also secondary to the acknowledged higher rates of obesity of the North American population, more specifically, the U.S. (17). In addition, an accurate appraisal of the radiation exposure faced by an urologist demands an incorporation of data from the entire spectrum of urology procedures performed using fluoroscopy, not just a subset of procedures such as PNLs. To our knowledge, this is the first published investigation to report cumulative radiation exposure data for a single urologist from a North American institution. It is also one of the only datasets that incorporates a number of different urological procedures, such as SWL, which have been excluded from many of the prior publications. This variety of endourological surgeries and procedures more accurately reflects an endourologist's practice, and may come closer to estimating true radiation exposure over a given time period. This is the only study to achieve a semblance of broad generalization and realistic application of such data. Our data presents a summation of exposures across a 9 month period as opposed to averaged doses of selected cases, allowing practitioners a more comprehensive reference standard for an understanding of radiation exposure.

Regarding study design, we incorporated a single TLD placed outside the thyroid shield, yielding mrem values for DDE, LDE, and SDE, giving a reasonably accurate estimate of total upper-body exposure radiation exposure. Although the study only utilized this singular location for placement of the TLD, this is thought to be consistent with the current practices of most North American urologists. We nevertheless acknowledge there are limitations - the study's applicability to individual urologists is limited by factors which may vary between practitioners, including operating facility and equipment, fellowship status, experience, and position in the operating room. In addition, inherent to any case series is a lack of randomization and controls, which limits our ability to account for differences in stone burden, surgical complexity, and patient body habitus. We also found that we could draw no significant correlations between increasing fluoroscopy time (minutes) and increasing radiation exposure (mrems). Although this would appear to make sense intuitively, the data did not yield such results. This could be secondary to any of the confounding factors listed above, and may also draw attention to TLDs as, perhaps, limited instruments in their ability to measure accurate radiation exposure. Such findings may deserve further review in future studies.

Using current devices and measures, our findings demonstrate that the quantity of radiation an academic urologist with a high-case volume is exposed to over the course of 9 months would appear to be below ICRP recommendations. Efforts to improve radiation safety, however, continue to be of utmost importance. The continued effort of the urologic community to reduce the fluoroscopy time required for a given procedure is essential. In conjunction with these efforts, we hope that our results will serve as a foundation for a reference standard for North American urologists from which they may extrapolate their respective radiation exposures. Importantly, we hope that such data will heighten awareness of radiation risk to practicing urologists in North America and encourage practitioners to continue safe radiation practices.

It remains essential to emphasize that there is no "safe" level of radiation exposure, and even small amounts could potentially cause a stochastic effect, such as cancer. This is why keeping the radiation dose as low as reasonably achievable (ALARA), a concept designated as optimization by the IRCP, is so essential to keep in mind during practice (9). Optimization requires indentifying parameters and using procedures/protocols to yield the necessary clinical information, while keeping radiation doses as low as possible (1).

\section{CONCLUSIONS}

Over a 9 month period, total radiation exposure for an endourology practice appears to be within accepted limits, as suggested by the IRCP (DDE 5000 mrem, LDE 15,000 mrem and SDE $50,000 \mathrm{mrem})$. Although fluoroscopy time did not correlate with radiation exposure, future prospec- 
tive studies can account for co-variates such as patient obesity and urologist distance from radiation source.

\section{CONFLICT OF INTEREST}

None declared.

\section{REFERENCES}

1. Linet MS, Slovis TL, Miller DL, Kleinerman R, Lee C, Rajaraman $P$, et al.: Cancer risks associated with external radiation from diagnostic imaging procedures. CA Cancer J Clin. 2012; Epub.

2. Ferrandino MN, Bagrodia A, Pierre SA, Scales CD Jr, Rampersaud E, Pearle MS, et al.: Radiation exposure in the acute and short-term management of urolithiasis at 2 academic centers. J Urol. 2009; 181: 668-72; discussion 673.

3. Mancini JG, Raymundo EM, Lipkin M, Zilberman D, Yong $D$, Bañez LL, et al.: Factors affecting patient radiation exposure during percutaneous nephrolithotomy. J Urol. 2010; 184: 2373-7.

4. Ngo TC, Macleod LC, Rosenstein DI, Reese JH, Shinghal R: Tracking intraoperative fluoroscopy utilization reduces radiation exposure during ureteroscopy. J Endourol. 2011; 25: 763-7.

5. Greene DJ, Tenggadjaja CF, Bowman RJ, Agarwal G, Ebrahimi KY, Baldwin DD: Comparison of a reduced radiation fluoroscopy protocol to conventional fluoroscopy during uncomplicated ureteroscopy. Urology. 2011; 78: 286-90.

6. Safak M, Olgar T, Bor D, Berkmen G, Gogus C: Radiation doses of patients and urologists during percutaneous nephrolithotomy. J Radiol Prot. 2009; 29: 409-15.
7. Elkoushy MA, Andonian S: Prevalence of orthopedic complaints among endourologists and their compliance with radiation safety measures. J Endourol. 2011; 25: 1609-13.

8. Liu SZ: Biological effects of low level exposures to ionizing radiation: theory and practice. Hum Exp Toxicol. 2010; 29: 275-81.

9. [No authors listed]: The 2007 Recommendations of the International Commission on Radiological Protection. ICRP publication 103. Ann ICRP. 2007; 37: 1-332.

10. Rao PN, Faulkner K, Sweeney JK, Asbury DL, Sambrook P, Blacklock NJ: Radiation dose to patient and staff during percutaneous nephrostolithotomy. Br J Urol. 1987; 59: 508-12.

11. Hellawell GO, Mutch SJ, Thevendran G, Wells E, Morgan RJ: Radiation exposure and the urologist: what are the risks? J Urol. 2005; 174: 948-52; discussion 952.

12. Kumari G, Kumar P, Wadhwa P, Aron M, Gupta NP, Dogra PN: Radiation exposure to the patient and operating room personnel during percutaneous nephrolithotomy. Int Urol Nephrol. 2006; 38: 207-10.

13. Majidpour HS: Risk of radiation exposure during PCNL. Urol J. 2010; 7: 87-9.

14. Lowe FC, Auster M, Beck TJ, Chang R, Marshall FF: Monitoring radiation exposure to medical personnel during percutaneous nephrolithotomy. Urology. 1986; 28: 221-6.

15. Giblin JG, Rubenstein J, Taylor A, Pahira J: Radiation risk to the urologist during endourologic procedures, and a new shield that reduces exposure. Urology. 1996; 48: 624-7.

16. Ritter M, Krombach P, Martinschek A, Siegel FP, Schmitt M, Weiss $\mathrm{C}$, et al.: Radiation exposure during endourologic procedures using over-the-table fluoroscopy sources. J Endourol. 2012; 26: 47-51.

17. Finucane MM, Stevens GA, Cowan MJ, Danaei G, Lin JK, Paciorek CJ, et.: National, regional, and global trends in body-mass index since 1980: systematic analysis of health examination surveys and epidemiological studies with 960 country-years and 9.1 million participants. Lancet. 2011; 377: 557-67.

\section{Correspondence address:} Dr. Roger L. Sur

U.C. San Diego Comprehensive Kidney Stone Center Department of Surgery and Division of Urology 200 W. Arbor Drive \#8897 San Diego, CA 92130-8897, USA

Fax: + 1619 543-6557 E-mail: rlsur@ucsd.edu 